\section{Secondary prevention in cognitive frailty: the Treviso Dementia Registry}

\author{
Maurizio Gallucci \\ Cognitive Impairment Center, Local \\ Health Authority 9 of Treviso, Treviso, \\ Italy
}

\begin{abstract}
Dementia is one of the most disabling health conditions for older people. Increasing attention is paid to the preclinical phase such as cognitive frailty and mild cognitive impairment, and to the prevention programs designed to reduce the number of patients in the future. The aims of this brief report are therefore: i) to illustrate an action plan currently active in Treviso and that is aimed at secondary prevention in cognitive frailty subjects on the Treviso Dementia (TREDEM) Registry; ii) to highlight the results achieved by the TREDEM Registry up to now and how these can be used in future research.
\end{abstract}

\section{Introduction}

Frailty is a multidimensional construct characterized by age-related increased vulnerability to stressors due to reduced capacity of various physiological systems. Frailty is closely associated with adverse health outcomes, especially in older people, including falls, disability, hospitalizations, and mortality. The most common definition of frailty was developed by Fried and colleagues on the basis of work in the Cardiovascular Health Study ${ }^{1}$ and the Women's Health and Aging Studies; ${ }^{2}$ this definition of frailty is limited to physical aspects. Even though the frailty construct, introduced by Fried and colleagues, is widely used and accepted by the scientific community, there is evidence of the importance of considering other common age-related conditions such as cognitive impairment. Recently, a consensus panel has proposed the identification of the so-called cognitive frailty as a heterogeneous clinical manifestation characterized by the simultaneous presence of both physical frailty and cognitive impairment. The key factors defining this condition include the presence of physical frailty and cognitive impairment (clinical dementia rating scale $=0.5$ ) and the exclusion of concurrent Alzheimer's disease (AD) dementia or other dementias. In particular Kelaiditi and colleagues underline that physical impairment and social isolation in older persons may explain a cognitive decline independent of a neurodegenerative condition. ${ }^{3}$ According to these considerations, there is a growing consensus to include cognitive impairment as a significant component of the operational definitions of frailty. ${ }^{4}$ Both cognitive and physical performance can be negatively influenced in parallel by factors such as chronic disease, sedentary lifestyles and poor socioeconomic conditions. ${ }^{5-10}$ Many studies have demonstrated that poor baseline physical performance results in cognitive impairment or dementia. ${ }^{11-14}$ Increasing evidence indicates, through prospective studies, that regular exercise is able to reduce the risk of mild cognitive impairment (MCI) and dementia. ${ }^{15}$ Neuroimaging studies of cognitive networks also show that regular exercise improves connectivity and increases neuroplasticity which translates into better learning skills. ${ }^{16}$ The American College of Sports Medicine recommends that exercise programs for older adults include both aerobic and non-aerobic physical activities, such as resistance training, balance training, and stretching for optimal general health. To elucidate the relationships between the concepts of cognitive frailty and healthy lifestyles in the light of the recent literature would be a fruitless exercise without producing practical initiatives. The aims of this brief report are therefore: i) to illustrate an action plan currently active in Treviso aimed at secondary prevention in cognitive frailty subjects on the Treviso Dementia (TREDEM) Registry; ii) to highlight the results achieved by the TREDEM Registry up to now and how these can be used in future research.

\section{Materials and Methods}

\section{The Treviso Dementia Registry}

The TREDEM Registry is an observational data collection on cognitive decline conducted at the Cognitive Impairment Center at the Local Health Authority no. 9 of Treviso (LHA9) and consists of data from 1364 subjects in the TREDEM study ${ }^{17-20}$ collected between 2000 and 2010 plus those collected later using the same methods; in total over then 2500 subjects up to now. The TREDEM Registry was approved by the Treviso Province Hospitals Ethics Committee. All participants are outpatients attending the Cognitive Impairment Center and undergo a multidimensional assessment consisting of socio-demographic and clinical (cognitive, behavioral, neurological, functional, physical) evaluation as already described in the TREDEM Study and Registry. ${ }^{17-22}$ All subjects undergo the standard laboratory and instrumental workup for cognitive impairment (including assessment of thyroid-stimulating hormone, vitamin B12, folate, and homocysteine concentrations, as well as brain comput-
Correspondence: Maurizio Gallucci, Cognitive Impairment Center, Local Health Authority 9 of Treviso, S. Maria di Ca' Foncello Hospital, Piazza Ospedale 1,31100 Treviso, Italy.

E-mail:mgallucci@ulss.tv.it

Key words: Cognitive frailty; secondary prevention; Treviso Dementia Registry.

Conflict of interest: the author declares no potential conflict of interest.

Received for publication: 5 June 2016. Accepted for publication: 4 July 2016.

This work is licensed under a Creative Commons Attribution-NonCommercial 4.0 International License (CC BY-NC 4.0).

(C) Copyright M. Gallucci, 2016

Licensee PAGEPress, Italy

Geriatric Care 2016; 2:6056

doi:10.4081/gc.2016.6056

ed tomography scan). Among other scales and questionnaires, the multidimensional prognostic index (MPI), based on a standardized comprehensive geriatric assessment has been systematically applied since 2008 when it was built by Alberto Pilotto. ${ }^{23}$ A questionnaire for the detection of frailty and disability realized by Cesari and colleagues is also administered to the participants to detect the characteristics of physical disability and frailty. ${ }^{24}$

\section{An action plan}

Camminando e leggendo... ricordo (CLR) (Walking and reading... I remember) is a practical initiative of secondary prevention built by the Cognitive Impairment Center and the Department of Prevention of LHA9 in Treviso and aimed at no-frail, pre-frail and frail MCI subjects. $^{22}$ The CLR physical exercise program we propose is composed of a two-phase group training with 30 min of walking at moderate intensity on a dirt path and 30 min of exercises for balance, flexibility and joint mobilization; resistance training with small equipment and weights; gait training with rapid changes of direction and speed variation. Each session ends with $7 \mathrm{~min}$ of stretching. The subjects who enroll in this multicomponent program group take part in the activities for 2 days/week under the supervision of a physical therapist and personal trainer. A bi-monthly reading group activity, led by experienced trainers, has also been added. This practice has the purpose of ensuring a cognitive stimulation: subjects are invited to comment on the readings and complete them at home. The combination of the two activities, always performed in the group, also offers an important opportunity for socialization. During the physical activity and reading meetings the trainers 
often recommend adherence to healthy lifestyles, to the Mediterranean diet and stopping smoking so as to promote a virtuous cycle of healthy behaviors. Given the success of this initiative, we have decided to monitor the participants over time together with a control group to carry out a pilot prospective observational study to support our model with scientific evidence. ${ }^{22}$ We propose, therefore, to assess the promotion of physical activity, reading and healthy lifestyles in non-frail, pre-frail and frail MCI subjects, to evaluate: i) the rate of conversion to dementia compared to that of the control group; ii) the level of functional autonomy over time in the conduct of basal and instrumental activities of daily living (ADL, IADL) $; 25,26$ iii) the quality of life of MCI subjects detected using an ad hoc questionnaire such as The psychological general well-being index. ${ }^{27,28}$ We received funding from the Center for the Control of Chronic Diseases of the Italian Ministry of Health to assess the results, effectiveness and transferability of our model. The local Ethic Committee approved the present CLR study design.

\section{Results from the Treviso Dementia Registry}

We measured homocysteinemia (Hcy) in subjects with $\mathrm{AD}$, with vascular dementia, and in healthy controls. Participants in the TREDEM group (constituting the dementia groups) presented significantly higher concentrations of Hcy than the controls, even after adjustment for potential confounders (i.e., age, albumin, serum folate and vitamin B12) ${ }^{29}$ Consistent findings were also reported in other of our reports suggesting Hcy as an important biological risk factor in the mechanisms underlying cerebral atrophy and cognitive decline..$^{19,30,31} \mathrm{We}$ also found close associations between low serum folate levels and severe cortical-subcortical atrophy along with severe hippocampal atrophy. ${ }^{19}$ We also described that the MPI correctly estimates the probability of death in cognitive impairment outpatients during a maximum observation period of approximately four years, and the probability of hospitalization for acute events in the twelve months following the date of evaluation. ${ }^{18}$

Another of our findings in TREDEM was that 3R mental stimulation can benefit cognitive functions, level of autonomy in ADL and it can also reduce caregiver distress. Behavioral disturbances were reduced even considering a main potential confounding factor such as treatment with anticholinesterase drugs. ${ }^{20}$ One of our recent studies shows that non-pharmacological and contextual factors, linkable to environmental enrichment and brain and cognitive reserve, may affect the long-term response to cholinesterase inhibitor treatment. ${ }^{21}$

\section{Discussion: future perspectives}

Our immediate challenges are: i) to continue the CLR case-control study to evaluate the effectiveness of the secondary prevention model we proposed; ii) to apply the frailty index to the TREDEM Registry sample. The frailty index was proposed and validated by Rockwood and colleagues in the Canadian Study of Health and Aging ${ }^{32}$ and may be an interesting tool in the field of cognitive impairment. The frailty index was designed on an arithmetical model of deficit accumulation; in the sense that, the more deficits a person has, the more the person will be frail..$^{33}$ The frailty index is composed of a checklist of non-predefined variables, i.e., deficits, constituted by signs, disabilities, diseases and laboratory findings. The frailty index is simply the ratio between deficits presented by the individual and the total number of deficits considered, thus providing a continuous measurement of frailty ranging between 0 , absence of frailty, and 1, maximum frailty. ${ }^{34}$ Estimates of risk have been reported to be robust when a minimum set of 50 heterogeneous and multidimensional variables are considered. We have identified fifty variables (Table 1), selected in the TREDEM Registry, in collaboration with Matteo Cesari and Marco Canevelli, so as to perform a multidimensional consideration of the overall health status of the aging individuals.

\section{Conclusions}

The field of cognitive impairment has often been characterized by mistrust, hopelessness, helplessness and even inevitability. The concept of prevention, finally also applied in the context of loss of memory, brings hope and trust. The Italian National Dementia Plan

Table 1. The 50 items composing the Treviso Dementia Registry frailty index.

\begin{tabular}{ll}
\hline 1. & Hypertension \\
2. & Ischemic heart disease \\
\hline 3. & Chronic heart failure \\
4. & Arrhythmia \\
\hline 5. & Peripheral arterial disease \\
6. & Diabetes \\
\hline 7. & Thyroid dysfunction \\
8. & Osteoporosis \\
\hline 9. & History of bone fractures \\
10. & Renal failure \\
\hline 11. & Parkinsonism \\
12. & History of stroke \\
13. & COPD \\
14. & Gastric disorder \\
\hline 15. & Intestinal disorder \\
\hline 16. & Cirrhosis \\
\hline 17. & Genitourinary disorder \\
\hline
\end{tabular}

18. Chronic venous insufficiency
19. Osteoarthritis
20. Cancer
21. Dyslipidemia
22. Hearing impairment
23. Vision impairment
24. Help eating (ADL)
25. Help toileting (ADL)
26. Incontinence (ADL)
27. Help transferring (ADL)
28. Help bathing (ADL)
29. Help dressing (ADL)
30. Help shopping (IADL)
31. Help taking medication (IADL)
32. Help using telephone (IADL)
33. Help with transportation (IADL)
34. Help with money (IADL)

35. Help with food preparation (IADL)
36. Help with housekeeping (IADL)
37. Help with laundry (IADL)
38. Depression $(\mathrm{GDS} \geq 10)$
39. Anxiety
40. Agitation $(\mathrm{NPI} \geq 4)$
41. Apathy $(\mathrm{NPI} \geq 4)$
42. Sleep disorders $(\mathrm{NPI} \geq 4)$
43. Appetite/eating disorders $(\mathrm{NPI} \geq 4)$
44. Hemoglobin $(<13.5 \mathrm{~g} / \mathrm{dL}$ in $\mathrm{males} ;<12.0 \mathrm{~g} / \mathrm{dL}$
in females $)$
45. Platelets $\left(<150 \times 10^{3} / \mathrm{mm}^{3}\right)$
46. White cells $\left(<4 \times 10^{3} / \mathrm{mm}^{3}\right)$
47. Albumin $(<3.5 \mathrm{~g} / \mathrm{dL})$
48. Creatinine $(<0.6 \mathrm{mg} / \mathrm{dL})$
49. Folic acid $\left(<2.7 \mathrm{ng} / \mathrm{mL}^{2}\right)$
50. Hypotension $(\mathrm{SBP}<90 \mathrm{mmHg})$

COPD, chronic obstructive pulmonary disease; ADL, basal activities of daily living; IADL, instrumental ADL; GDS, geriatric depression scale; NPI, neuropsychiatric inventory; SBP, systolic blood pressure. 
places prevention in the foreground, recommending primary and secondary prevention strategies. ${ }^{35}$

Preclinical cognitive changes may predict an increased risk of dementia and select some subgroups as possible targets for preventive or therapeutic interventions such as physical exercise. ${ }^{36}$

The TREDEM Registry database is rich in multi-dimensional data which is useful for gaining greater and deeper knowledge and understanding of the frail status within the framework of cognitive impairment.

\section{References}

1. Fried L, Tangen C, Walston J, et al. Frailty in older adults. Evidence for a phenotype. J Gerontol A Biol Sci Med Sci 2001;56:M14657.

2. Bandeen-Roche K, Xue Q, Ferrucci L, et al. Phenotype of frailty. Characterization in the women's health and aging studies. J Gerontol A Biol Sci Med Sci 2006;61:262-6.

3. Kelaiditi E, Cesari M, Canevelli M, et al. Cognitive frailty: rational and definition from an (I.A.N.A./.A.G.G.) international consensus group. J Nutr Health Aging 2013;17:726-34.

4. Van Kan G, Rolland Y, Bergman H, et al. The I.A.N.A. task force on frailty assessment of older people in clinical practice. $\mathrm{J}$ Nutr Health Aging 2008;12:29-37.

5. Anstey K, Smith G. Interrelationships among biological markers of aging, health, activity, acculturation, and cognitive performance in late adulthood. Psychol Aging 1999;14:605-18.

6. Guralnik JM, Ferrucci L, Penninx BW, et al. New and worsening conditions and change in physical and cognitive performance during weekly evaluations over 6 months. The Women's Health and Aging Study. J Gerontol A Biol Sci Med Sci 1999;54:M410-22.

7. Kuh D, Bassey E, Butterworth S, et al. Grip strength, postural control, and functional leg power in a representative cohort of British men and women. Associations with physical activity, health status, and socioeconomic conditions. J Gerontol A Biol Sci Med Sci 2005;60:224-31.

8. Richards M. Cognitive links across the lifecourse and implications for health in later life. Age Ageing 2000;29:477-8.

9. Richards M, Sacker A. Lifetime antecedents of cognitive reserve. J Clin Exp Neuropsychol 2003;25:614-24.

10. Alfaro-Acha A, Al Snih S, Raji M, et al. Does 8-foot walk time predict cognitive decline in older Mexicans Americans? J Am Geriatr Soc 2007;55:245-51.
11. Angevaren M, Aufdemkampe G, Verhaar H, et al. Physical activity and enhanced fitness to improve cognitive function in older people without known cognitive impairment Cochrane Database Syst Rev 2008;16:CD005381.

12. Teri L, Logsdon R, McCurry S. Exercise interventions for dementia and cognitive impairmen. The Seattle Protocols. J Nutr Health Aging 2008;12:391-4.

13. Geda YE, Roberts RO, Knopman DS, et al. Physical exercise, aging, and mild cognitive impairment: a population-based study. Arch Neurol 2010;67:80-6.

14. Liu-Ambrose T, Eng JJ, Boyd LA, et al. Promotion of the mind through exercise (PROMoTE): a proof-of-concept randomized controlled trial of aerobic exercise training in older adults with vascular cognitive impairment. BMC Neurol 2010;10:14.

15. Ahlskog J, Geda Y, Graff-Radford N, Petersen R. Physical exercise as a preventive or disease-modifying treatment of dementia and brain aging. Mayo Clin Proc 2011;86:876-84.

16. Erickson K, Kramer A. Aerobic exercise effects on cognitive and neural plasticity in older adults. Br J Sports Med 2008;43:22-4.

17. Gallucci M, Mariotti E, Saraggi D, et al. The Treviso Dementia (TREDEM) Study: a biomedical, neuroradiological, neuropsychological and social investigation of dementia in North-Eastern Italy. J Frailty Aging 2012;1:24-31.

18. Gallucci M, Battistella G, Bergamelli C, et al. Multidimensional prognostic index (MPI) in an outpatient setting: mortality and hospitalizations. The Treviso Dementia (TREDEM) study. J Alzheimers Dis 2014;42:1461-8.

19. Gallucci M, Zanardo A, Bendini M, et al. Serum folate, homocysteine, brain atrophy, and auto-CM system: the Treviso Dementia (TREDEM) study. J Alzheimers Dis 2014;38:581-7.

20. Spagnolo $\mathrm{P}$, Aricò $\mathrm{M}$, Bergamelli $\mathrm{C}$, et al. $\mathrm{A}$ pilot study on behavioral and cognitive effects of cognitive stimulation training based on $3 \mathrm{R}$ mental stimulation. The Treviso Dementia (TREDEM) study. Neurorehabilitation 2015;36:151-6.

21. Gallucci M, Spagnolo P, Aricò M, Grossi E. Predictors of response to long-term cholinesterase inhibitors treatment of Alzheimer disease: data mining from TREDEM Registry. J Alzheimers Dis 2016;50: 969-79.

22. Gallucci M, Mazzetto M, Spagnolo P, et al. Mild cognitive impairment, from theory to practical intervention: "Camminando e leggendo... ricordo" (Walking and reading... I remember), an action plan. The Treviso Dementia (TREDEM) registry. Ann Ist Super Sanità 2016;52:240-8.
23. Pilotto A, Ferrucci L, Franceschi M, et al. Development and validation of a multidimensional prognostic index for one-year mortality from comprehensive geriatric assessment in hospitalized older patients. Rejuvenation Res 2008;11:151-61.

24. Cesari M, Demougeot L, Boccalon H, Vellas B. Prevalence of frailty and mobility limitation in a rural setting in France. J Frailty Aging 2012;1:169-73.

25. Katz S. Studies of illness in the aged. JAMA 1963;185: 914.

26. Lawton M, Brody E. Assessment of older people. Selfmaintaining and instrumental activities of daily living. Gerontologist 1969;9:179-86.

27. Carotenuto A, Fasanaro AM, Molino I, et al. The psychological general well-being index (PGWBI) for assessing stress of seafarers on board merchant ships. Int Marit Health 2013;64:215-20.

28. Lundgren-Nilsson Å, Jonsdottir I, Ahlborg G, Tennant A. Construct validity of the psychological general well being index (PGWBI) in a sample of patients undergoing treatment for stress-related exhaustion: a rasch analysis. Health Qual Life Outcomes 2013;11:2.

29. Gallucci M, Zanardo A, De Valentin L, Vianello A. Homocysteine in Alzheimer disease and vascular dementia. Arch Gerontol Geriatr 2004;9:195-200.

30. Folin M, Baiguera S, Gallucci M, et al. A cross-sectional study of homocysteine-, nolevels, and CT-findings in Alzheimer dementia, vascular dementia and controls. Biogerontology 2005;6:255-60.

31. Baiguera S, Gallucci M, Zanardo A, Folin M. Effect of homocysteine and nitric oxide levels on specific computed axial tomography measurements in Alzheimer disease. CEJB 2007;2:99-108.

32. Rockwood K, Song X, MacKnight C, et al. A global clinical measure of fitness and frailty in elderly people. CMAJ 2005;173:489-95.

33. Mitnitski AB, Mogilner AJ, Rockwood K. Accumulation of deficits as a proxy measure of aging. Sci World J 2001;1:323-36.

34. Searle SD, Mitnitski A, Gahbauer EA, et al. A standard procedure for creating a frailty index. BMC Geriatr 2008;8:24.

35. Di Fiandra T, Canevelli M, Di Pucchio A, et al. The Italian Dementia National Plan. Ann Ist Super Sanità 2015;51:261-4.

36. Di Carlo A, Baldereschi M, Lamassa M, et al. Daily function as predictor of dementia in cognitive impairment, no dementia (CIND) and mild cognitive impairment (MCI): an 8-year follow-up in the ILSA study. J Alzheimers Dis 2016;53:505-15. 